\title{
Systemic administration of ghrelin did not restore angiogenesis in hindlimb ischemia in control and diet-induced obese mice
}

\author{
Tahergorabi $\mathrm{Z}^{1}$, Khazaei $\mathrm{M}^{2}$, Rashidi $\mathrm{B}^{3}$ \\ Department of Physiology, Faculty of Medicine, Mashhad University of Medical Sciences, Mashhad, Iran. \\ khazaei@med.mui.ac.ir
}

\begin{abstract}
Background: Ghrelin is a novel growth hormone releasing peptide that mainly regulates food intake and energy homeostasis, however, recently, it is indicated that it may be closely related with physiological and/ or pathological angiogenesis.

Objectives: The objective of the present study was to evaluate the effect of systemic ghrelin administration on angiogenesis in hindlimb ischemia in normal and diet-induced obese mice.

Methods: 24 male C57BL/6 mice were fed with high-fat diet (HFD) or standard for 14 weeks. Then, the mice underwent unilateral hindlimb ischemia. Next, each group was divided into the two subgroups: treatment with ghrelin $(100 \mu \mathrm{g} / \mathrm{kg}$, twice daily, Sc) or without treatment. After 10 days, the animals were sacrificed, blood samples were taken and the gastrocnemius muscles removed.

Results: There was no significant difference in capillary/fiber ratio in hind limb ischemia between obese and control groups. Administration of ghrelin reduced serum nitric oxide (NO) and leptin and increased vascular endothelial growth factor (VEGF) concentrations in obese mice, however, did not change the capillary/fiber ratio in ischemic legs.

Conclusion: Systemic administration of ghrelin did not restore angiogenesis in hindlimb ischemia in control and diet-induced obese mice (Fig. 4, Ref. 35). Text in PDF www.elis.sk.

Key words: obesity, angiogenesis, ghrelin, hindlimb ischemia, leptin.
\end{abstract}

Obesity, a complex metabolic disorder, is created following a prolonged dysbalance of caloric intake and energy expenditure (1) and is associated with the most chronic and severe human diseases including type 2 diabetes, hypertension, cardiovascular diseases, stroke and certain types of cancer such as colorectal, breast and prostate cancers (2).

During positive energy balance, there is an increased number and size of white adipocytes, that is dependent critically to angiogenesis (3). Blood vessels by providing nutrients and oxygen, growth factors and cytokines in plasma supply growth and survival signals in adipocytes to mainten their physiological functions (4).

Angiogenesis, that is the formation new blood vessels from preexisting ones, spatially and temporally is associated with adipogenesis (5). Angiogenesis is involved in a series of physiological and pathological process including embryonic development, wound healing, chronic inflammation, tumor progression and

${ }^{1}$ Department of Physiology, Birjand University of Medical Sciences, Birjand, Iran, ${ }^{2}$ Department of Physiology, Faculty of Medicine, Mashhad University of Medical Sciences, Mashhad, Iran, and ${ }^{3}$ Department of Anatomy, Isfahan University of Medical Sciences, Isfahan, Iran

Address for correspondence: M Khazaei, MD, Department of Physiology, Faculty of Medicine, Mashhad University of Medical Sciences, Mashhad, Iran

Phone and Fax: +985138009227

Acknowledgment: The authors would like to thank the vice chancellor research of Isfahan University of Medical Sciences for their financial support (Research project No.189142). revascularization of the myocardium following myocardial infarction (MI)(6). Increase in adipocyte size lead to hypoxia in adipose tissue and induction of transcription factor of hypoxia inducible factor (HIF-1) (7). This in turn through binding to the hypoxia response element (HRE) of target genes such as VEGF (vascular endothelial growth factor) and angiopoietin 2 induce an angiogenic response (8).

Ghrelin is a novel growth hormone released mainly from the stomach (9). It is involved in food intake, regulation of appetite and energy homeostasis, in addition modulation of gastrointestinal, cardiovascular, pulmonary and immune functions, cell proliferation/apoptosis (10). A number of in vivo and in vitro studies have reported that it can be as a pro-angiogenic or anti-angiogenic factor and thus may be closely related with angiogenesis (11).

Hindlimb ischemia is a clinical manifestation of peripheral arterial disease (PAD) (12). Since obesity and type 2 diabetes are considered as two of the strongest risk factors for the development of PAD (13), the objective of present study was to evaluate the effect of systemic ghrelin administration on angiogenesis in hindlimb ischemia in normal and diet-induced obese mice.

\section{Materials and methods}

Animals

24 male mice C57BL/6J, 20-30 g, 5 weeks old obtained from Pasteur Institute of Iran. They were housed on a 12 h light / 12 $\mathrm{h}$ dark cycle at $25^{\circ} \mathrm{C}$ room temperature with free access to food 
$35-40$

and water ad libitum. The animals were acclimatized to the laboratory conditions for 7 days and then, randomly assigned into the two groups: obese and control $(n=12$ each). The ethical committee of the Isfahan University of Medical Sciences approved all study protocol.

\section{Animal diets}

Obesity was induced by feeding of the mice with high-fat diet (HFD) (labratories BioServ, Cat \#F3282, USA) (14) included 59 $\%$ fat, $27 \%$ carbohydrate, $14 \%$ protein for 14 weeks. The control groups received standard diet (Pasteur Institute, Iran). Body weights of the animals were monitored weekly.

\section{Hindlimb ischemia model and treatment groups}

After 14 weeks, the mice underwent unilateral hindlimb ischemia surgery. For this purpose, the mice were anaesthized by intraperitoneal injection of ketamin $(75 \mathrm{mg} / \mathrm{kg})$ and xylazine $(7.5 \mathrm{mg} /$ $\mathrm{kg}$ ). Then, an incision was made in the skin overlying the middle portion of the left hindlimb. The femoral artery and its associated branches and vein were isolated, ligated and excised (15). The animals were followed up for next $48 \mathrm{~h}$. Then, the mice were randomly assigned to one of the following experimental groups: control and obese groups treated with ghrelin, control and obese groups received vehicle ( $n=6$ each group). Ghrelin was injected subcutaneously, twice daily, at the dose of $100 \mu \mathrm{g} / \mathrm{kg}$ (Tocris Co. Bristol, UK) for 10 days (16). Then, the animals were sacrificed,
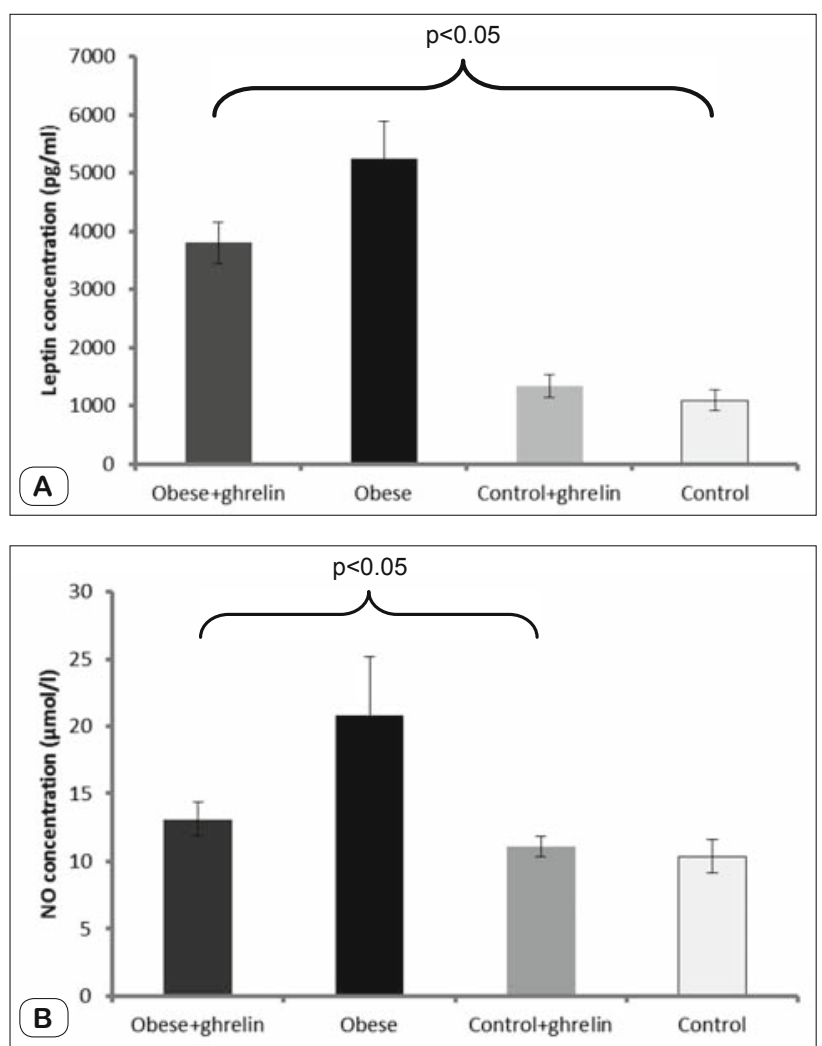

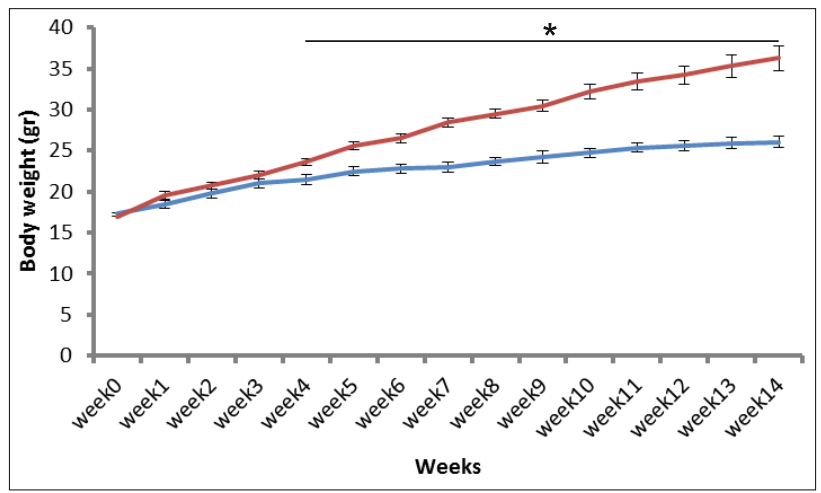

Fig. 1. Body weights in the obese and control groups. Values are expressed as Mean \pm SEM; ${ }^{*} \mathbf{p}<0.05$ compare to control.

blood samples were taken and the gastrocnemius muscles harvested for immunohistochemical staining.

\section{Biochemical analysis}

Blood samples were centrifuged for 30 minutes. The serums were removed and stored at less than $-20^{\circ} \mathrm{C}$ for subsequent analysis. The serum levels were measured in each case by sandwich enzyme immunoassay using specifically available kits. Mouse VEGF and soluble form of VEGF receptor-1 (sVEGFR1) kits (R\&D systems, Minneapolis, MN, USA), mouse Leptin kit (Invitrogen, Camarillo, CA 93012), mouse Insulin kit (Mercodia,

Fig. 2. Serum leptin (A), NO (B), VEGF (C) and sVEGFR-1(D) concentrations in obese and control groups. Ghrelin administration reduced serum NO and leptin and increased serum VEGF level in obese mice (E). Data are shown as mean \pm SEM. ${ }^{*}$ p $<0.05$ compare to control. 

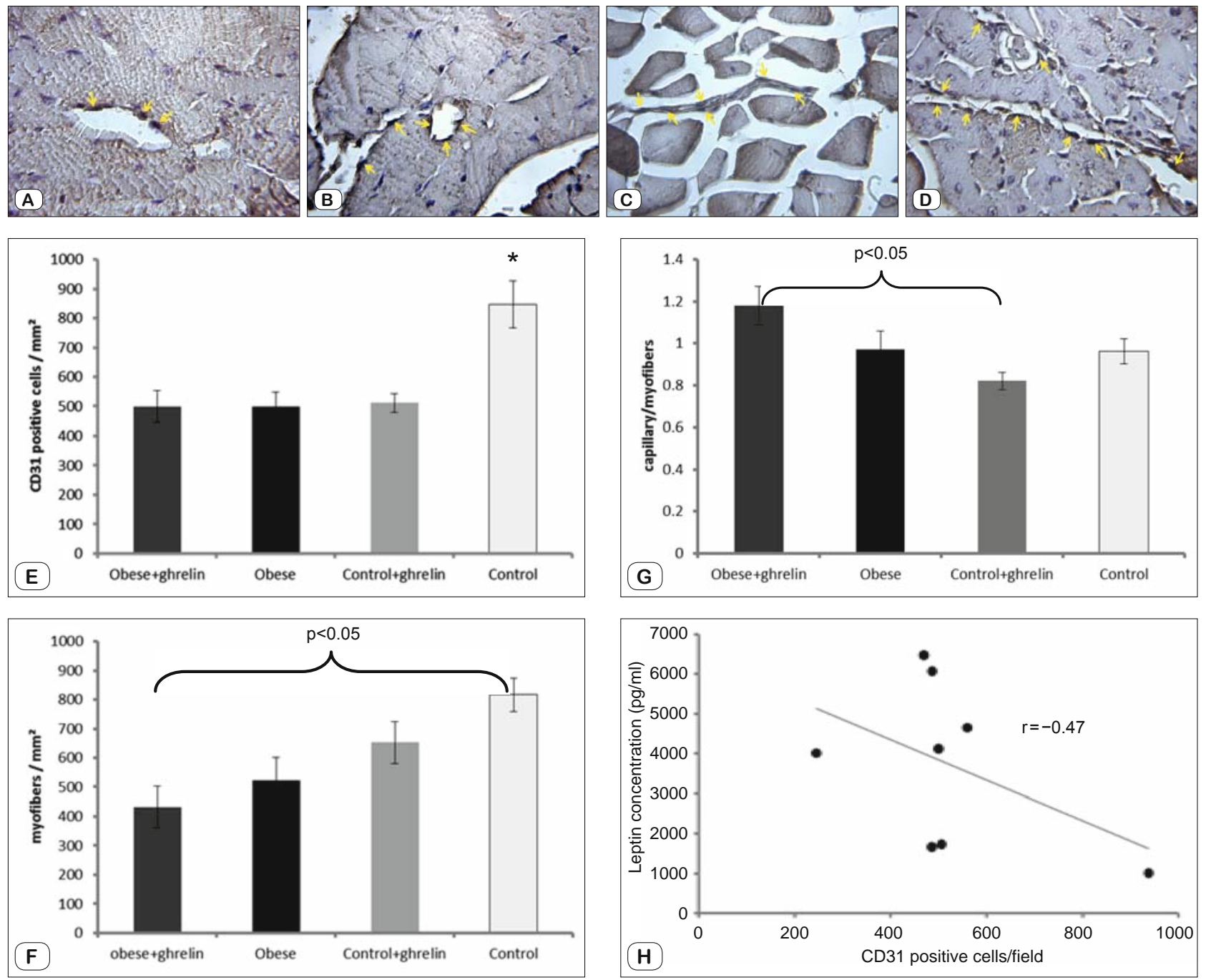

Fig. 3. Effect of HFD and ghrelin on angiogenesis in ischemic legs. The histological sections were stained with immunohistochemical technique. A - obese+ghrelin; B - obese; C - control+ghrelin; D - control. Ghrelin administration did not change the number of CD31 positive cells and capillary/fiber ratio in obese and control groups (F, G). Arrows indicates the CD31 positive cells. Data are shown as mean \pm SEM ( $n=6$ each group). Correlation between the number of CD31 positive cells/field in ischemic skeletal muscle and serum leptin concentration $(r=-0.47)(H)$.

Uppsala, Sweden) and serum nitrite, the main metabolite of nitric oxide (NO) (Promega Corp, USA) were measured according to the manufacturer's instructions. The minimum sensitivity of VEGF and sVEGFR-1 assays were $3.9 \mathrm{pg} / \mathrm{ml}$ and $3.8 \mathrm{pg} / \mathrm{ml}$, respectively with an intra- and interassay coefficients of variation less than 10 $\%$ and $5 \%$, respectively. The limit of detection of serum nitrite and insulin concentration is $2.5 \mu \mathrm{M}$ and $\leq 0.2 \mu \mathrm{g} / \mathrm{l}$ respectively. The minimum detectable dose of mouse leptin is $<50 \mathrm{pg} / \mathrm{ml}$.

\section{Capillary density analysis}

Tissue samples were obtained from gastrocnemius muscles on postoperative day 10 and put in formalin $10 \%$ solution. Fixed gastrocnemius muscles were paraffin-embedded and cut into $4 \mu \mathrm{m}$ thickness and applied on silanized glass slides for immunohistochemical assays. Endothelial cells were identified by using primary antibody (rabbit anti-mouse CD31; 1:50; Abcam Co.). The angiogenesis was expressed as the numbers of CD31-positive cells and capillary/fiber ratio, which were counted using an Olympus light microscope at $\times$ 40 magnifications in five different fields for each skeletal muscle.

\section{TUNEL assay}

Apoptosis in skeletal muscle was detected using TUNEL staining (terminal deoxynucleotidyl-transferase-mediated dUTP nick end labeling) (Roche, Germany). Tissue slides were deparaffinized and treated with proteinase $\mathrm{K}(20 \mu \mathrm{g} / \mathrm{ml})$. Then, the slides were incubated with terminal deoxynucleotidyl transferase (TdT) for 1 hour also, peroxidase antibody for 30 minutes at 37 ${ }^{\circ} \mathrm{C}$. During the TUNEL procedure, tissue slides were washed in PBS (phosphate-buffer saline) ( $\mathrm{pH}$ 7.4). Finally, TUNEL positive cells were counted in 5 different fields for each mouse using an Olympus light microscope at $\times 40$ magnification. The apoptosis was expressed as the TUNEL positive nuclei per field. 


\section{Statistical analysis}

All values were expressed as the mean \pm S.E.M. The statistical software SPSS version 16 was used for data analysis. The significance of differences between groups was assessed with one-way ANOVA. Correlation analysis was examined using the Pearson's correlation coefficient. $\mathrm{p}$ value less than 0.05 was considered statistically significant.

\section{Results}

\section{Body weight}

Body weights were measured at the beginning of the experiment (week 0 ) and every week for 14 weeks. The body weight in the obese group was significantly higher than control $(\mathrm{p}<0.05)$ (Fig. 1).

\section{Blood glucose and serum insulin}

Blood glucose level in diet-induced obese mice was significantly higher than in the control groups $(\mathrm{p}<0.05)$. Ghrelin treatment
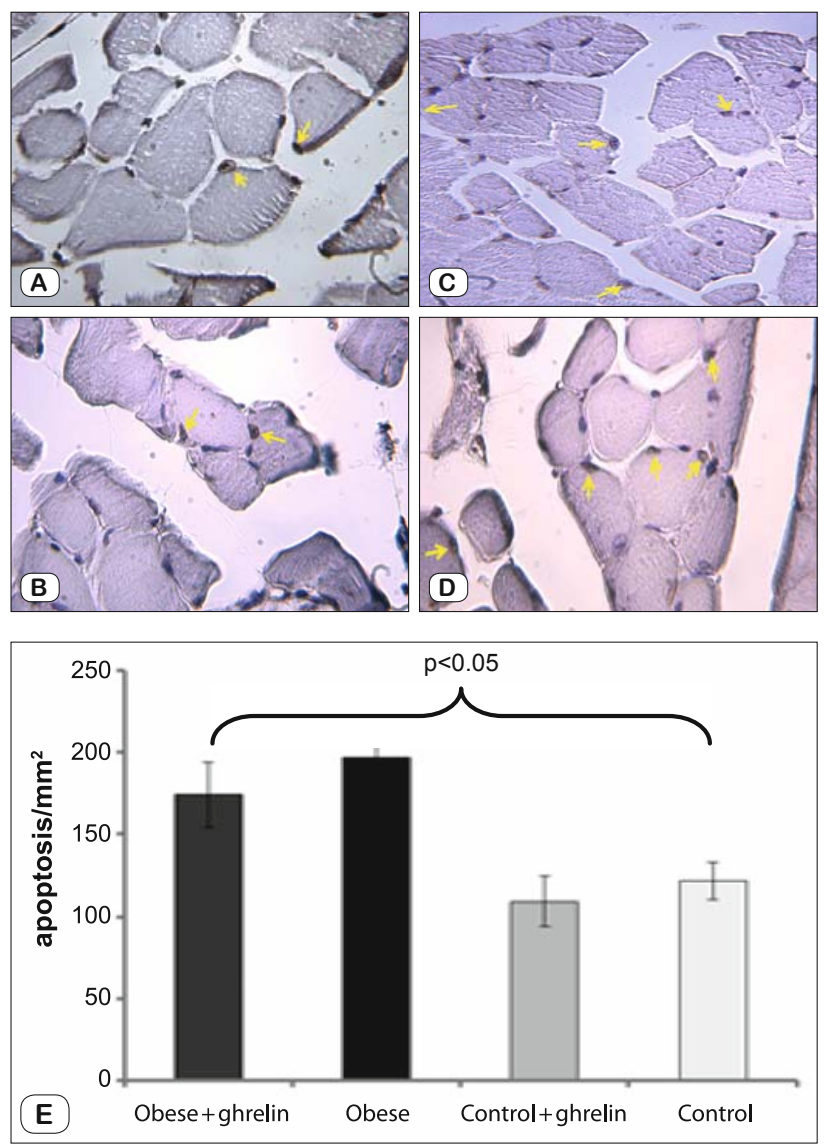

Fig. 4. Effect of ghrelin on apoptosis in the hindlimb ischemia. The histological sections were stained with TUNEL method. A- obese+ghrelin; B - obese; C - control+ghrelin; D - control. HFD loading significantly increased apoptosis in the skeletal muscle and ghrelin administration did not change apoptosis in obese and control groups (E). Arrows indicates the apoptosis. Data are shown as the mean \pm SEM $(n=6$ each group). did not change blood glucose level in obese and control groups ( $p$ $>0.05$ ). There was significant difference in insulin concentration between control and obese mice. Ghrelin decreased serum insulin levels in obese group $(\mathrm{p}<0.05)$ without significantly changes in control animals.

Serum biomarkers of angiogenesis: effect of ghrelin

HFD produced higher serum leptin levels compared to control animals $(\mathrm{p}<0.05)$ (Fig. 2 A). Serum nitrite level in obese animals was significantly higher than control mice $(\mathrm{p}<0.05)$ (Fig. 2 B), while, serum VEGF and sVEGFR1 concentrations were not different between obese and control groups ( $p>0.05)$ (Fig. 2 C, D). Treatment with ghrelin increased serum VEGF and decreased serum $\mathrm{NO}$ and leptin levels in obese mice $(\mathrm{p}<0.05)$.

\section{Angiogenesis in hindlimb ischemia muscle: effect of ghrelin}

Quantitative analysis of CD31 positive cells in hindlimb ischemic muscles by immunohistochemistry showed that ghrelin administration did not change the number of CD31 positive cells in obese group, however reduced in control mice $(p<0.05)$ and did not alter the number of myofibers $(p>0.05)$ (Fig. 3 E, F). However, since during hindlimb ischemia muscle atrophy or edema is a common phenomenon (17), we evaluated the capillary/fiber ratio and there were no differences between obese and control groups ( $p$ $>0.05$ ). Ghrelin administration did not change the capillary/fiber ratio in obese and control groups $(p>0.05)$ (Fig. $3 \mathrm{G})$. Samples of immunohistochemical staining were presented in Figures $3 \mathrm{~A}-\mathrm{D}$.

\section{Correlation analysis}

Scatter plots in Figure $3 \mathrm{H}$ presents a negative correlation between the number of CD31 positive cells in the ischemic skeletal muscle and serum leptin levels $(r=-0.47, p=0.23)$.

\section{Apoptosis after hindlimb ischemia: effect of ghrelin}

There was an increased apoptosis in the ischemic muscle of obese mice compared to that in the control group as determined by TUNEL staining $(p<0.05)$. Ghrelin treatment did not alter apoptosis in the ischemic muscle in obese and control group ( $p>$ 0.05 ) (Fig. 4 E). Samples of immunohistochemical staining were presented in Figures 4 A-D.

\section{Discussion}

This study examined the effect of systemic ghrelin administration on angiogenesis in hindlimb ischemia in normal and dietinduced obese mice.

We demonstrated that HFD decreased the number of CD31 positive cells in ischemic skeletal muscle, however, capillary/myofibers ratio was not different between obese and control groups. On the other hand, measurement of the serum parameters exhibited that obese animals had higher serum NO and leptin levels than that control mice.

Leptin is an adipocyte derived hormone substantially involved in the weight regulation by influencing the food intake and energy expenditure. Several studies have reported that it also has a direct pro-angiogenic activity (18). NO, the key regulator of vascular 
and metabolic homeostasis, is a product of an enzymatic reaction catalyzed by one of the three isoforms of NO synthase (NOS) (19). Leptin is an adipocyte derived hormone and its serum levels are correlated with body mass index (BMI) (20). Several studies have shown that HFD led to hyperleptinemia and leptin resistance (21). Obesity is associated with chronic low grade inflammation condition (22) and leptin can induce the iNOS (inducible nitric oxide synthase) expression and NO production in inflammatory conditions such as obesity (23). Also, HFD causes upregulation of inflammatory cytokines such as iNOS and ROS (reactive oxygen species) and down regulation of superoxide dismutase (SOD) that in turn can lead to impaired vasodilatation and reduced sensitivity to vasoconstrictors (24) that possibly can be involved in impaired angiogenic response in obesity.

We also demonstrated that HFD produced a higher blood glucose and serum insulin levels than the control group. HFD with upregulation of ROS can cause a progression of insulin resistance (25). Evidence shows ROS effects on vascular function is dose dependent. Under physiological condition, it modulates pro-angiogenic pathway and higher levels of ROS impairs neovascularization (26). In obese mice, onset of hyperleptinemia occurs prior to that hyperinsulinemia and insulin resistance (27) and a number of studies have demonstrated that murine leptin can increase insulin secretion (28). Insulin resistance in turn can lead to impaired neovascularization and APC (angiogenic progenitor cell) mobilization (29) that possibly can be involved in impaired angiogenic response in obesity.

Accordingly, the capillary rarefaction in our study reduced the number of myofibers in ischemic muscle of obese mice that possibly could in consequence increase the apoptosis in obese mice and involve an impaired angiogenesis. One of the obesity complications is an ectopic accumulation of lipids in non-adipose tissue such as skeletal muscle that occurs in consequence of increased adipose tissue lipolysis, it is termed lipotoxicity. It also can lead to cell dysfunction or cell death (30). Furthermore, obesity is associated with chronic low-grade inflammation condition and activation of inflammatory mediators and signaling pathways that can lead to apoptosis in vascular cells of the skeletal muscle.

In the correlation analysis, we found a negative correlation between the number of CD31 positive cells in the skeletal muscle and serum leptin levels that confirmed the negative effect of leptin on angiogenesis in the skeletal muscle in obese mice.

We also showed that ghrelin treatment could not change the capillary/myofibers ratio in ischemic legs of obese and control group. In addition, ghrelin administration did not alter apoptosis in obese and control groups. The biochemical analysis revealed that ghrelin could increase serum VEGF and decrease NO, leptin and insulin levels in obese mice.

Ghrelin a peptide of 28 amino acids is recognized as an important factor for stimulation of appetite and increased food intake. In addition, a number of studies have showed it could be a pro-angiogenic or anti-angiogenic factor and maight be closely related with angiogenesis $(31,32)$.

VEGF is a major angiogenic factor that most of the angiogenic activity in adipose tissue attributed to (33) possibly; there was an impaired signaling despite of elevated VEGF levels by ghrelin that could not alter angiogenic response following ischemia in obese group. In addition, in obesity, there is an extensive inflammatory response and expression of many inflammatory mediators. Leptin exerts proinflammatory effects and ghrelin dose-dependently inhibits proinflammatory mediators. Thus, ghrelin and leptin have mutually antagonistic effects on inflammatory cytokine expression in inflammatory condition such as obesity (34) and could be involved in angiogenic response

Serum ghrelin levels correlated negatively with insulin concentrations in obesity and insulin resistance that in several studies have been demonstrated. Acylated ghrelin administration possibly causes downregulation of insulin and improvement of insulin sensitivity in obese mice (35) .

Based on our knowledge, no study has been done to evaluate the effect of systemic ghrelin administration on angiogenesis in hindlimb ischemic model and we found that despite the changes in some biomarkers of angiogenesis, systemic administration of ghrelin did not restore angiogenesis in hindlimb ischemia in control and diet-induced obese mice.

\section{References}

1. Item F, Konrad D. Visceral fat and metabolic inflammation: the portal theory revisited. Obes Rev 2012; 13 (Suppl 2): 30-39.

2. Cao Y. Angiogenesis modulates adipogenesis and obesity. J Clin Invest 2007; 117 (9): 2362-2368.

3. Cho CH, Koh YJ, Han J, Sung HK, Jong Lee H, Morisada T et al. Angiogenic role of LYVE-1-positive macrophages in adipose tissue. Circ Res 2007; 100 (4): e47-e57.

4. Cao Y. Adipose tissue angiogenesis as a therapeutic target for obesity and metabolic diseases. Nat Rev Drug Discov 2010; 9 (2): 107-115.

5. Crandall DL, Hausman GJ, Kral JG. A review of the microcirculation of adipose tissue: anatomic, metabolic, and angiogenic perspectives. Microcirculation 1997; 4 (2): 211-232.

6. Carmeliet P. Angiogenesis in health and disease. Nat Med 2003; 9 (6): 653-660.

7. Wang B, Wood IS, Trayhurn P. Dysregulation of the expression and secretion of inflammation-related adipokines by hypoxia in human adipocytes. Pflugers Arch 2007; 455 (3): 479-492.

8. Halberg N, Khan T, Trujillo ME, Wernstedt-Asterholm I, Attie AD, Sherwani S et al. Hypoxia-inducible factor 1alpha induces fibrosis and insulin resistance in white adipose tissue. Mol Cell Biol 2009; 29 (16): 4467-4483.

9. Rocha-Sousa A, Saraiva J, Henriques-Coelho T, Falcao-Reis F, Correia-Pinto J, Leite-Moreira AF.Ghrelin as a novel locally produced relaxing peptide of the iris sphincter and dilator muscles. Exp Eye Res 2006; 83 (5): 1179-1187.

10. Leite-Moreira AF, Soares JB. Physiological, pathological and potential therapeutic roles of ghrelin. Drug Discov Today 2007; 12: 276-288.

11. Conconi MT, Nico B, Guidolin D, Baiguera S, Spinazzi R, Rebuffat $\mathbf{P}$ et al. Ghrelin inhibits FGF-2-mediated angiogenesis in vitro and in vivo. Peptides 2004; 25 (12): 2179-2185. 
12. Waters RE, Terjung RL, Peters KG, Annex BH. Preclinical models of human peripheral arterial occlusive disease: implications for investigation of therapeutic agents. J Appl Physiol (1985) 2004; 97 (2): 773-780.

13. Beckman JA, Creager MA, Libby P. Diabetes and atherosclerosis: epidemiology, pathophysiology, and management. JAMA 2002; 287 (19): 2570-2581.

14. Peyot ML, Pepin E, Lamontagne J, Latour MG, Zarrouki B, Lussier $\mathbf{R}$ et al. Beta-cell failure in diet-induced obese mice stratified according to body weight gain: secretory dysfunction and altered islet lipid metabolism without steatosis or reduced beta-cell mass. Diabetes 2010; 59 (9): 2178-2187.

15. Li P, Shibata R, Maruyama S, Kondo M, Ohashi K, Ouchi N et al. Fenofibrate promotesischemia-induced revascularization through the adiponectin-dependent pathway. Am J Physiol Endocrinol Metab 2010; 299 (4): E560-E566.

16. Li L, Zhang LK, Pang YZ, Pan CS, Qi YF, Chen L et al. Cardioprotective effects of ghrelin and des-octanoyl ghrelin on myocardial injury induced by isoproterenol in rats. Acta Pharmacol Sin 2006; 27 (5): 527-535.

17. Couffinhal T, Dufourcq P, Barandon L, Leroux L, Duplaa C. Mouse models to study angiogenesis in the context of cardiovascular diseases. Front Biosci 2009; 14: 3310-3325.

18. Liu L, Meydani M. Angiogenesis inhibitors may regulate adiposity. Nutr Rev 2003; 61 (11): 384-387.

19. Choi JW, Pai SH, Kim SK, Ito M, Park CS, Cha YN. Increases in nitric oxide concentrations correlate strongly with body fat in obese humans. Clin Chem 2001; 47 (6): 1106-1109.

20. Hou N, Luo JD. Leptin and cardiovascular diseases. Clin Exp Pharmacol Physiol 201138 (12): 905-913.

21. Dyck DJ. Leptin sensitivity in skeletal muscle is modulated by diet and exercise. Exerc Sport Sci Rev 2005; 33 (4): 189-194.

22. Wellen KE, Hotamisligil GS. Inflammation, stress, and diabetes. J Clin Invest 2005; 115 (5): 1111-1119.

23. Vuolteenaho K, Koskinen A, Kukkonen M, Nieminen R, Päivärinta $\mathbf{U}$, Moilanen T et al. Leptinenhances synthesis of proinflammatory mediators in human osteoarthritic cartilage mediator role of $\mathrm{NO}$ in leptin-induced PGE2, IL-6, and IL-8 production. Mediators Inflamm 2009; 2009: 345838. doi: $10.1155 / 2009 / 345838$.
24. Sweazea KL, Walker BR. High fat feeding impairs endothelin-1 mediated vasoconstriction through increased iNOS-derived nitric oxide. Horm Metab Res 2011; 43 (7): 470-476.

25. Kolluru GK, Bir SC, Kevil CG. Endothelial dysfunction and diabetes: effects on angiogenesis, vascular remodeling, and wound healing. Int J Vasc Med 2012; 2012: 918267. Doi 10.1155/2012/918267.

26. Griendling KK, FitzGerald GA. Oxidative stress and cardiovascular injury: Part I: basic mechanisms and in vivo monitoring of ROS. Circulation 2003; 108 (16): 1912-1916.

27. Jiang L, Wang Q, Yu Y, Zhao F, Huang P, Zeng R et al. Leptin contributes to the adaptive responses of mice to high-fat diet intake through suppressing the lipogenic pathway. PLoS One 2009; 4 (9): e6884. doi: 10.1371/journal.pone.0006884.

28. Shimizu H, Ohtani K, Tsuchiya T, Takahashi H, Uehara Y, Sato N et al. Leptin stimulatesinsulin secretion and synthesis in HIT-T 15 cells. Peptides 1997;18(8):1263-1266.

29. Kahn MB, Yuldasheva NY, Cubbon RM, Smith J, Rashid ST, Viswambharan $\mathbf{H}$ et al. Insulin resistance impairs circulating angiogenic progenitor cell function and delays endothelial regeneration. Diabetes 2011; 60 (4): 1295-1303.

30. Turpin SM, Ryall JG, Southgate R, Darby I, Hevener AL, Febbraio MAet al. Examinationof'lipotoxicity' in skeletal muscle of high-fat fed and ob/ob mice. J Physiol 2009; 587 (Pt7): 1593-1605.

31. Wang L, Chen Q, Li G, Ke D. Ghrelin stimulates angiogenesis via GHSR1a-dependentMEK/ERK and PI3K/Akt signal pathways in rat cardiac microvascular endothelial cells. Peptides 2012; 33 (1): 92-100.

32. Sato T, Ida T, Nakamura Y, Shiimura Y, Kangawa K, Kojima M. Physiological roles of ghrelin on obesity. Obesity research \& clinical practice 2013. In press.

33. Lijnen HR. Angiogenesis and obesity. Cardiovasc Res 2008; 78 (2): 286-293.

34. Dixit VD, Schaffer EM, Pyle RS, Collins GD, Sakthivel SK, Palaniappan $\mathbf{R}$ et al. Ghrelin inhibits leptin- and activation-induced proinflammatory cytokine expression by human monocytes and $\mathrm{T}$ cells. J Clin Invest 2004; 114 (1): 57-66.

35. Pulkkinen L, Ukkola O, Kolehmainen M, Uusitupa M. Ghrelin in diabetes and metabolic syndrome. Int J Pept 2010; 2010. doi. org/10.1155/2010/248948. 